\title{
The association between smoking and the prevalence of metabolic syndrome and its components in patients with psoriasis aged 30 to 49 years
}

\author{
Agnieszka B. Owczarczyk-Saczonek ${ }^{1}$, Roman Nowicki² \\ ${ }^{1}$ Department of Dermatology, Municipal Hospital, Olsztyn, Poland \\ Head of the Department: Prof. Waldemar Placek MD, PhD \\ 2Department of Dermatology, Venereology and Allergology, Gdansk Medical University, Gdansk, Poland \\ Head of the Department: Prof. Roman Nowicki MD, PhD
}

Postep Derm Alergol 2015; XXXII (5): 331-336 DOI: $10.5114 /$ pdia.2015.54743

\begin{abstract}
Introduction: Cigarette smoking may exacerbate and cause psoriasis. Moreover, smokers are more likely to develop insulin resistance and metabolic syndrome (MS).

Aim: To assess the prevalence of MS and its components in patients with psoriasis, who smoke, compared with the general Polish population of smokers.

Material and methods: We studied 29 patients with psoriasis (female $=9$, male $=20$ ), smokers, aged 30 to 49 years. Metabolic syndrome and its components were assessed using the IDF definition and compared to the results obtained in a representative sample of adult Poles in the NATPOL 2011 study in the same age group, including smokers. Results: The results have shown that patients with psoriasis are more likely to be smokers $(p<0.0034)$ and the frequency of smoking in men is approximately $25 \%$ higher than in males of the control group $(p<0.0017)$. The prevalence of MS in patients with psoriasis who smoke was $27.58 \%$ and in the control group $25.2 \%(p>0.05)$. Mean body mass index was $26.07 \mathrm{~kg} / \mathrm{m}^{2}$ in psoriasis patients and $25.59 \mathrm{~kg} / \mathrm{m}^{2}$ in the control group $(p>0.05)$, and abdominal obesity was $88.82 \mathrm{~cm}$ and $90.02 \mathrm{~cm}(p>0.05)$, respectively. There were no differences in hypertension (34.48\% vs. $31.6 \%, p<0.05)$ and mean HOMA-IR (1.80 vs. 1.77, $p>0.05)$. In lipid parameters, the differences were observed only in women with psoriasis - higher levels of HDL, triglycerides and ApoB/ApoA1 index compared with addicted women in the control group.

Conclusions: Men with psoriasis are more often addicted to smoking. Women with psoriasis who smoke have often disturbances of the lipid profile.
\end{abstract}

Key words: psoriasis, metabolic syndrome, insulin resistance, smoking.

\section{Introduction}

The presence of the same genetic predisposition is not sufficient for the development of psoriasis. Provocative factors that initiate a cascade of immune disorders play an important role in the formation of lesions.

Cigarette smoking is one of the factors that may exacerbate and cause psoriasis. The risk of psoriasis is higher in present and former smokers compared with those who never smoked [1-3]. Some studies confirm the relationship between smoking intensity and the severity of psoriasis [3, 4]. Smoking releases large amounts of free radicals and is a cause of "oxidative stress" $[1,3,5]$.
The free radicals stimulate, regardless of cytokines, cell signalling pathways active in psoriasis including mitogen-activated protein kinase (MAPK), nuclear factor-кB (NF-кB), Janus kinase and JAK-STAT pathway [5]. Moreover, nicotine stimulates dendritic cells, macrophages and keratinocytes. They release cytokines, mainly tumor necrosis factor- $\alpha$ (TNF- $\alpha$ ), IL-6, activating the T-cells and sustaining the course of chronic psoriasis $[1,5,6]$. Interleukin-6, in turn, helps to increase the production of C-reactive protein (CPR) in liver, which can lead to systemic inflammation [6] and contribute to the onset of metabolic disorders. Nicotine can also modulate the functional capacity of dendritic cells as antigen present-

Address for correspondence: Agnieszka B. Owczarczyk-Saczonek MD, Department of Dermatology, Municipal Hospital, 30 Wojska Polskiego St, 10-595 Olsztyn, Poland, phone: +48 601057 800, fax: +48 8967866 75, e-mail: aganek@wp.pl Received: 22.07.2013, accepted: 2.01.2014. 
ing cells (APC) by an increased expression of costimulatory molecules (CD86, CD40), adhesion molecules (LFA-1, CD54), and the expression of major histocompatibility complex class II (MCH-II) [1, 2, 4]. Furthermore, cigarette smoking increases the expression of genes predisposing to psoriasis, including HLA-Cw6, HLA-DQA1*0201 and CYP1A1 [5] and smokers with HLA-Cw6 have an 11 times greater risk of developing psoriasis compared to nonsmokers without HLA-Cw6 [7].

Cigarette smoking, besides hypertension and hypercholesterolemia, is a major risk factor for cardiovascular disease [8]. It is the cause of severity of inflammation, endothelial dysfunction, atherogenic dyslipidaemia and coagulation disorders, resulting in the initiation and progression of atherosclerosis [9]. Smoking, inter alia, increases leukocyte count, and serum concentration of CRP, IL- 6 and TNF- $\alpha$ [9].

According to the concept of the 'psoriatic march', which has been presented in recent years, psoriasis, obesity and smoking cause inflammation which influences the rapid development of atherosclerosis in patients [10] Therefore, an attempt was made to assess the impact of psoriasis on prevalence of metabolic disorders in smokers.

\section{Aim}

The aim of this study was to assess the impact of cigarette smoking on metabolic syndrome (MS) and its components: insulin resistance, atherogenic dyslipidaemia, abdominal obesity and hypertension in patients with psoriasis aged 30 to 49 years, compared to Poles, who participated in the NATPOL 2011 study in the same age group.

\section{Material and methods}

The study included 62 patients with psoriasis aged 30 to 49 years, including 29 smokers (9 women and 20 men) treated at the out-patient clinic and the Department of Dermatology, Municipal Hospital in Olsztyn. Psoriatic patients with chronic and acute inflammatory diseases other than psoriasis, cancer, a history of cardiovascular complications and heart, kidney or liver failure $(n=1)$ were excluded from the study.

The control group consisted of 861 people of the Polish population aged between 30 and 49 years, including 250 smokers (101 women and 149 men) with a negative history of psoriasis. These people were included in the NATPOL research project in 2011 conducted by a team from the Hypertension and Diabetes Clinic, University Clinical Centre in Gdansk. The project involved the study of a representative group of 2400 Polish citizens in 20102011 and the prevalence of hypertension and other risk factors for cardiovascular disease, including lipid disor- ders and diabetes, among these people were estimated. A detailed description of the sampling will be published in "Polish Cardiology".

It has been assumed that smoking $\geq 5$ cigarettes per day is an addiction.

The following parameters were estimated in all subjects: body mass index (BMI), waist circumference measured in centimetres [11], blood pressure, according to the Polish Society of Hypertension guidelines 2003. The dermatological examination assessed the severity of psoriasis - PASI [12].

The patients had laboratory tests done under fasting conditions, $12 \mathrm{~h}$ after the last meal, evaluating the lipids ( $\mathrm{HDL}$, triglycerides, apolipoprotein Al and B) and carbohydrate metabolism (glucose, insulin) and CRP. Insulin resistance index HOMA-IR (homeostasis model assessmentinsulin resistance) was calculated [13] and to evaluate lipids, ApoB/ApoA1 indexes were estimated [14].

Metabolic syndrome was diagnosed using the International Diabetes Federation (IDF) criteria modified in 2009 [15], in which abdominal obesity (for Europeans: $\geq 94 \mathrm{~cm}$ for men, $\geq 80 \mathrm{~cm}$ for women) is not a prerequisite for the diagnosis, but it is one of the five criteria. The diagnosis of MS is about meeting three of five criteria (abdominal obesity, triglycerides level $\geq 150 \mathrm{mg} / \mathrm{dl}$ or treatment of hypertriglyceridaemia, $\mathrm{HDL}<40 \mathrm{mg} / \mathrm{dl}$ in men and $<50 \mathrm{mg} / \mathrm{dl}$ in women, blood glucose $\geq 100 \mathrm{mg} / \mathrm{dl}$ or pharmacotherapy of hyperglycaemia and blood pressure: systolic $\geq 130 \mathrm{~mm} \mathrm{Hg}$ and/or diastolic blood pressure $\geq 85 \mathrm{~mm} \mathrm{Hg}$ or antihypertensive drug treatment) [16].

\section{Statistical analysis}

Statistical analysis of the results was performed using the statistical software package StatisticaPL v. 10. Analysis of variance and correlation was used to verify the hypotheses.

\section{Results}

Assessment of smoking in the study group compared to the NATPOL 2011 control group

The results showed that patients with psoriasis are more likely to smoke cigarettes compared to the NATPOL 2011 control group. These differences were also found considering patients' gender: the frequency of smoking is $25 \%$ higher in males with psoriasis (Table 1 ).

\section{Correlation between the severity of psoriasis (PASI) and cigarette smoking}

Correlation analysis of PASI (with and without considering patients' gender) and smoking showed no significant correlation between them. However, there was a significant correlation between smokers and alcohol drinkers. 
Table 1. Comparison of smoking frequency between patients with psoriasis and NATPOL 2011

\begin{tabular}{lcccccc}
\hline Variable & \multicolumn{2}{c}{ Total } & \multicolumn{2}{c}{ Female } & Male \\
\cline { 2 - 6 } & Study group & NATPOL & Study group & NATPOL & Study group & NATPOL \\
\hline $\begin{array}{l}\text { The number } \\
\text { of distinguished people }\end{array}$ & 29 & 250 & 9 & 101 & 20 & 149 \\
\hline Ratio & 0.4670 & 0.2900 & 0.3210 & 0.2510 & 0.5880 & 0.3240 \\
\hline Probability $(p)$ & \multicolumn{2}{c}{0.0034} & \multicolumn{2}{c}{0.0978} & 0.0017 \\
\hline
\end{tabular}

Note: If $p<\alpha=0.05$ - statistically significant differences.

\section{Evaluation of the prevalence of metabolic} syndrome and its components in smokers

The results showed no statistically significant difference between smokers from the NATPOL 2011 group and a group of patients with psoriasis in the incidence of MS. The prevalence of MS in smokers with psoriasis was $27.58 \%$ and for the control group $25.2 \%(p>0.05)$.

There were no differences either between the mean $\mathrm{BMI}$ and waist circumference in both groups, considering patients' gender. Mean BMI was $26.07 \mathrm{~kg} / \mathrm{m}^{2}$ in patients with psoriasis and $25.59 \mathrm{~kg} / \mathrm{m}^{2}$ for the control group $(p>0.05)$. Abdominal obesity was $88.82 \mathrm{~cm}$ and $90.02 \mathrm{~cm}$ ( $p>0.05)$, respectively.

There were no differences between the mean HOMA-IR and diabetes in both groups, even after considering patients' gender. The mean HOMA-IR was 1.93 in patients with psoriasis and 1.94 for the control group ( $p>0.05$ ). Lipid analysis showed no differences between the mean triglycerides, ApoA1, ApoB, and ApoB/ApoA1 indexes in patients with psoriasis compared to the NATPOL 2011 control group. However, there were statistically significant differences after considering the patients' gender. Women with psoriasis who smoke had a higher HDL level, triglycerides and ApoB/ApoA1 index in comparison to the smoking women from the NATPOL 2011 control group.

There were no statistically significant differences in the prevalence of MS criteria for blood pressure (> 130/85 $\mathrm{mm} \mathrm{Hg}$ ) between a group of smokers with psoriasis and the control group (34.48\% vs. $31.6 \%$, $p<0.05)$.

\section{Discussion}

\section{Incidence of smoking in psoriatic patients}

Many studies have confirmed the association between smoking and psoriasis, especially pustular psoriasis. A study conducted by Naldi et al. Has shown that one in 5 cases of psoriasis has been associated with smoking. Nicotine addiction may initiate psoriasis in genetically predisposed individuals and influences the disease severity. The risk increases with the number of cigarettes smoked daily and is higher in women than men: it is about 2.5 to 3.3 times higher among women who smoke more than 20 cigarettes per day and about
1.7 times higher among smoking men $[1,5]$. Li et al. assessed the correlation between smoking and the prevalence of psoriasis in the U.S. Population (Nurses' Health Study). Smokers, who smoked 1-14 cigarettes per day, had a relative risk of psoriasis of 1.81, those who smoked 1524 cigarettes per day had 2.04, and those who smoked 25 or more - up to 2.29 [17]. The authors have also observed a gradual disease risk reduction after smoking cessation. The risk of psoriasis was particularly increased in heavy smokers and those with long-term addiction and it was similar for both genders [17]. However, there are also controversial reports. Chinese research showed that smoking is a risk factor for psoriasis only among men [18].

Our own research showed a higher percentage of people addicted to smoking among patients with psoriasis, especially in males ( $25 \%$ higher). This can confirm the aforesaid effect of smoking on psoriasis [1, 5, 19].

An interesting observation in our study is the frequent coexistence of smoking habit and alcohol consumption in patients with psoriasis. In the literature, alcohol consumption has been described as a factor responsible for triggering psoriasis, but it is said that smoking increases the risk of the onset of the disease [20]. Naldi says that smokers who drink are twice as likely to develop the disease as non-smokers and non-drinkers [2].

It should be noted that psoriasis can have an impact on self-esteem and suffering from the disease can cause stress and the patients are more likely to turn to alcohol and cigarettes [19].

\section{Effect of cigarette smoking on metabolic syndrome and its components}

Many studies have shown that smoking increases the risk of developing MS. Nakanishi et al. found that the risk of developing MS was from 1.07 to 1.66 higher for smokers than non-smokers and it depended on the number of cigarettes smoked [18, 21, 22]. And smoking has an effect on metabolic disorders up to 20 years after quitting [21, 22]. However, there are also controversial reports. Polish studies by Chlebus et al. found a negative correlation between the prevalence of the MS and addictions [23].

Results of the studies by Gisondi to evaluate associations between psoriasis, MS and smoking are also incon- 
clusive. It was found that although people with psoriasis were more likely to smoke, the association between MS and psoriasis was not related to smoking [24].

\section{Impact of smoking on obesity}

It is believed that smoking can control weight. This mechanism is explained by the influence of nicotine on the increase in the resting metabolic rate and decreased appetite [22, 25]. Smoking 24 cigarettes per day increases energy expenditure by $10 \%$. However, the comparison of waist circumference or abdominal obesity shows different results, and smokers were found to have the largest waist circumference [22, 25].

This phenomenon can be explained by the influence of nicotine on insulin resistance, predisposing to obesity and endocrine dysfunction among smokers. Long-lasting elevated catecholamines and growth hormone levels decrease the number of insulin receptors and lead to pancreatic cells toxicity $[22,25]$.

Moreover, visceral adipose tissue is sensitive to cortisol, which is elevated in the serum of smokers because of an increased sympathetic nervous system activity. Furthermore, the elevation in testosterone and low oestrogen levels lead to the increase in visceral obesity in menopausal women. Women who smoke have lower levels of bioavailable oestrogen [22]. The substances found in tobacco smoke also damage the granulosa cells of ovaries, causing a decrease in oestrogen and an increase in testosterone [26], resulting in abdominal obesity.

There is a lot of controversy about it. Mizuno et al. found no differences in waist circumference among nonobese patients, smokers or non-smokers. However, the waist circumference was significantly larger in obese patients who smoked compared to non-smokers [27].

Our own research showed no differences between the group of patients and the NATPOL group, considering patients' gender.

\section{Effect of smoking on blood glucose and insulin resistance}

Numerous studies have shown that smoking reduces insulin sensitivity and causes type 2 diabetes and the longer the duration the higher the risk [22, 25, 27]. The study by Manson showed that the risk of diabetes increased with the number of cigarettes smoked per day: 20 cigarettes per day increased the risk by $50 \%$, and more than 20 up to $70 \%$ [28]. Smoking cessation improves insulin sensitivity, despite weight gain.

From 5 to 10 years after quitting smoking it is the same as in people who have never smoked [22, 28]. The Physicians' Health Study, which analysed over 22 thousand male physicians aged 40-84 who were addicted to smoking, showed that type 2 diabetes is more than twice as common in smokers as in non-smokers [29]. A similar correlation was found in the Nurse Health Study. The risk of developing diabetes was 1.4 for women who smoked more than 25 cigarettes per day [25].

There are reports which do not confirm such a correlation. The study by Godsland et al. showed that smokers had lower insulin levels after an oral glucose load than non-smokers [30]. Similar results were obtained by Szurkowska et al. identifying lower insulin levels in the fasting state and after a glucose load in smokers. Also Masulli et al. found no effect of smoking on insulin resistance [23].

Our own research found no differences between the average glucose values and HOMA-IR among the psoriatic patients and the NATPOL group, considering patients' gender.

\section{Impact of tobacco smoking on lipid metabolism}

Smoking modifies the lipid profile, leading to an increase in plasma levels of triglycerides (TG) and lower levels of HDL cholesterol [6, 21, 31-34]. Moreover, this correlation seems to be dependent on the number of smoked cigarettes [31]. Nicotine stimulates processes of lipolysis, leading to an increase in free fatty acids [21]. This reduces lipoprotein lipase activity and increases 3-hydroxy-3-methylglutaryl-CoA and glucose-6-phosphatase, which leads to lipid disorders [21]. In addition, there is an increased lipid oxidation and circulating lipid peroxidation products and antibodies against ox-LDL are significantly elevated in smokers. It is common knowledge that $\mathrm{Ox}-\mathrm{LDL}$ are antigens initiating the process of plaque formation [6].

The results have shown higher levels of HDL cholesterol in smoking women with psoriasis than in the control group, but also higher levels of triglycerides and ApoB/ApoA1. No such differences were seen among nonsmokers.

\section{Impact of tobacco smoking on hypertension}

The effect of smoking on blood pressure is also controversial. Frati et al. showed that blood pressure and heart rate increase during smoking, but this effect disappears within an hour [21]. Moreover, Weitzman et al. found that active smoking was associated with a lowering of blood pressure [6, 34]. Mild reduction in blood pressure in smokers may be related to decreased body weight [35]. This observation is supported by the weight gain and increased blood pressure among former smokers versus that observed among people who never smoked [35]. A vasodilator effect of cotinine, the major metabolite of nicotine, also may help to lower blood pressure [34].

According to other reports, the incidence of hypertension is increased among those who smoke 15 or more cigarettes per day [36]. Chronic smoking causes arterial stiffness, which may persist for several years after smoking cessation [36]. Hypertension among smokers may be related to chronic sympathetic nervous system activity of 
muscles in the arterial wall [36]. However, Geslain-Biquez et al. believe that there are no significant differences between smokers and non-smokers in the prevalence of hypertension [37].

Our own research showed no statistically significant differences in the prevalence of hypertension in smokers between the study group and the NATPOL group.

\section{Conclusions}

Cigarette smoking among male patients with psoriasis is $25 \%$ higher than in the general Polish population. Our study confirms that cigarette smoking may be a provoking factor for psoriasis, especially in men. Women with psoriasis who smoke had higher levels of HDL cholesterol, triglycerides and ApoA1/ApoB index than smoking women in the Polish population. There were no differences in the prevalence of the MS, obesity, insulin resistance and hypertension between patients with psoriasis who smoke and the Polish population.

\section{Acknowledgments}

We thank dr hab. n. med. Tomasz Zdrojewski, prof. dr hab. n. med. Bogdan Wyrzykowski, dr n. med. Piotr Bandosz, dr n. med. Marcin Rutkowski of the Department of Hypertension and Diabetology, Medical University in Gdansk, for sharing their data from the NATPOL 2011 Survey.

\section{Conflict of interest}

The authors declare no conflict of interest.

\section{References}

1. Naldi L, Mercuri SR. Smoking and psoriasis: from epidemiology to pathomechanisms. J Invest Dermatol 2009; 129: 2741-3.

2. Naldi L, Chatenoud L, Linder D, et al. Cigarette smoking, body mass index, and stressful life events as risk factors for psoriasis: results from an Italian case-control study. J Invest Dermatol 2005; 125: 61-7.

3. Szramka-Pawlak B, Kowalczyk MJ, Żaba R. Smoking conducive to falling ill with psoriasis - myth or reality? In: Psychological and medical aspects of skin diseases. Rzepa T, Szepietowski J, Żaba R (eds). Cornetis, Wroclaw 2011; 65-9. 37.

4. Wakkee M, Thio HB, Prens EP, et al. Unfavorable cardiovascular risk profiles in untreated and treated psoriasis patients. Atherosclerosis 2007; 190: 1-9.

5. Armstrong AW, Armstrong EJ, Fuller EN, et al. Smoking and pathogenesis of psoriasis. Br J Dermatol 2011; 165: 1162-8.

6. Ambrose JA, Barua RS. The pathophysiology of cigarette smoking and cardiovascular disease. J Am Coll Cardiol 2004; 43: 1731-7.

7. Jin Y, Zhang F, Yang S, et al. Combined effects of HLA-Cw6, body mass index and waist-hip ratio on psoriasis vulgaris in Chinese Han population. J Dermatol Sci 2008; 52: 123-9.
8. Zdrojewski T, Główczyńska R, Opolski G. Risk factors of cardiovascular diseases of Polish adult population - summary. In: Heart disease and vessels. Guide for GP. Opolski G, Lukas W, Steciwko A (eds). Via Medica, Gdańsk 2007; 283-90.

9. Urban M, Głowińska-Olszewska B, Krasowska I. Traditional risk factors for atherosclerosis. In: Atherosclerosis in children and youth. Urban M (ed.). Cornetis, Wrocław 2007; 76-120.

10. Boehncke WH, Boehncke S, Tobin AM, et al. The "psoriatic march": a concept of how severe psoriasis may drive cardiovascular comorbidity. Exp Dermatol 2011; 20: 303-7.

11. Zahorska-Markiewicz B, Podolec P, Kopeć G, et al. Guidelines of Polish Forum for Prevention on overweight and obesity. In: Handbook of Polish Forum for Prevention. Vol. 2. Podolec P (ed). Medycyna Praktyczna, Kraków 2010; 199-200.

12. Gottlieb AB, Chaudhari U, Baker DG, et al. The National Psoriasis Foundation Psoriasis Score System versus the Psoriasis Area Severity Index and Physician's Global Assessment: a comparison. J Drugs Dermatol 2003; 2: 260-6.

13. Szurkowska M, Szafraniec K, Gilis-Januszewska A, et al. The indicators of insulin resistance in the study population and their predictive value in determining the metabolic syndrome. Przegl Epidemiol 2005; 59: 743-51.

14. Brookes $L$. The apo B/A-I ratio - a stronger predictor of cardiovascular events than LDL, HDL, or total cholesterol, triglycerides, or lipid ratios. Medscape Cardiology 2006. Available at: http://www.medscape.com/viewarticle/541799.

15. Wyrzykowski B. Definitions of metabolic syndrome. In: Metabolic syndrome in clinical practice Wyrzykowski B (ed.). Via Medica, Gdańsk 2010; 114-38.

16. Alberti KGMM, Eckel RH, Grundy SM, et al. Harmonizing the metabolic syndrome. Circulation 2009; 120: 1640-5.

17. Li W, Han J, Choi HK, et al. Smoking and risk of incident psoriasis among women and men in the United States: a combined analysis. Am J Epidemiol 2012; 175: 402-13.

18. Nakanishi N, Takatorige T, Suzuki K. Cigarette smoking and the risk of the metabolic syndrome in middle-aged Japanese men and women: the JPHC Study Cohort I. Eur J CardiovasC Prevent Rehabil 2005; 13: 207-13.

19. Luty-Frąckiewicz A. The effect of smoking and drinking alcohol to lesions in patients with psoriasis. Przegl Dermatol 2003; 90: 275-80.

20. Huerta C, Rivero E, Garcia Rodriguez LA. Incidence and risk factors for psoriasis in the general population. Arch Dermatol 2007; 143: 1559-65.

21. Frati AC, Iniestra F, Ariza CR. Acute effect of cigarette smoking on glucose tolerance and other cardiovascular risk factors. Diabetes Care 1996; 19: 112-8.

22. Wierzejewska R, Jarosz $M$. Is smoking effective in weight control - review of the literature. Przegl Lek 2008; 65: 692-4.

23. Masulli M, Riccardi G, Galasso R, Vaccaro O. Relationship between smoking habits and the features of the metabolic syndrome in a nondiabetic population. Nutr Metab Cardiovasc Dis 2006; 16: 364-70.

24. Gisondi P, Tessari G, Conti A, et al. Prevalence of metabolic syndrome in patients with psoriasis: a hospital-based casecontrol study. Br J Dermatol 2007; 157: 68-73.

25. Szurkowska M. Influence of lifestyle on the levels of insulin in healthy people. In: The insulin levels in the metabolic syndrome. Szybiński Z (ed.). Wyd. Medyczne, Kraków 2003; 109-26.

26. Reich A, Straszak J, Śliwińska J. Analysis of the nicotinic addiction among patients with skin diseases. Dermatol Klin 2011; 13: 13-8. 
27. Mizuno O, Okamoto K, Sawada M, et al. Obesity and smoking: relationship with waist circumference and obesityrelated disorders in men undergoing a health screening. J Atheroscler Thromb 2005; 12: 199-204.

28. Manson JE, Ajani UA, Liu S, et al. A prospective study of cigarette smoking and the incidence of diabetes mellitus among US male physicians. Am J Med 2000; 109: 538-42.

29. Wannamethee SG, Shaper AG, Whincup PH, et al. Smoking cessation and the risk of stroke in middle-aged men. JAMA 1995; 274: 155-60.

30. Godsland IF, Leyva F, Walton C, et al. Associations of smoking, alcohol and physical activity with risk factors for coronary heart disease and diabetes in the first follow-up cohort of the Heart Disease and Diabetes Risk Indicators in a Screened Cohort study (HDDRISC-1). J Intern Med 1998; 244: 33-41.

31. Criqui MH, Wallace RB, Heiss G, et al. Cigarette smoking and plasma high-density lipoprotein cholesterol. The Lipid Research Clinics Program Prevalence Study. Circulation 1980; 62: 70-6.

32. Hering D, Kucharska W, Kara T, et al. Smoking is associated with chronic sympatetic activation in hypertension. Blood Press 2010; 19: 152-5.

33. Herron MD, Hinckley M, Hoffman MS, et al. Impact of obesity and smoking on psoriasis presentation and management. Arch Dermatol 2005; 141: 1527-34.

34. Weitzman M, Cook S, Auinger P, et al. Tobacco smoke exposure is associated with the metabolic syndrome in adolescents. Pediatr Cardiol Circ 2005; 112: 862-9.

35. Perkins KA, Epstein LH, Marks BL, et al. The effect of nicotine on energy expenditure during light physical activity. N Eng J Med 1989; 320: 898-903.

36. Bowman TS, Gaziano JM, Buring JE, et al. A prospective study of cigarette smoking and risk of incident hypertension in women. J Am Coll Cardiol 2007; 50: 2085-92.

37. Geslain-Biquez C, Vol S, Tichet J, et al. The metabolic syndrome in smokers. The D.E.S.I.R. study. Diabetes Metab 2003; 29: 226-34. 